\title{
Phenomenology of the Minimal Inverse-Seesaw with Abelian Flavour Symmetries
}

\author{
H. B. Câmara, ${ }^{1}$ R. G. Felipe, ${ }^{1,2}$ and F. R. Joaquim ${ }^{1, \S}$ \\ ${ }^{1}$ Departamento de Física and CFTP, Instituto Superior Técnico, Universidade de Lisboa, 1049-001 Lisboa, Portugal \\ ${ }^{2}$ ISEL - Instituto Superior de Engenharia de Lisboa, Instituto Politécnico de Lisboa, Rua Conselheiro Emídio Navarro, \\ 1959-007 Lisboa, Portugal
}

\begin{abstract}
We study the phenomenology of the minimal inverse-seesaw model composed of two "right-handed neutrinos" and two sterile singlet fermions, besides the Standard Model (SM) particle content. The model is supplemented with Abelian flavour symmetries to ensure maximal predictability and establish the most restrictive flavour patterns which can be realised by those symmetries. This setup requires the addition of a second scalar doublet and two complex scalar singlets to the SM enabling us to implement spontaneous CP violation. Such CP-violating effects can be successfully communicated to the lepton sector by means of the scalar singlets couplings with the new sterile fermions. The Majorana and Dirac CP phases are correlated, and the active-sterile neutrino mixing is fully determined by the active neutrino masses, mixing angles and $\mathrm{CP}$ phases. We investigate the constraints imposed on the model by the current experimental limits as well as future projected sensitivities on charged lepton flavour-violating decays and searches sensitive to the presence of heavy sterile neutrinos.
\end{abstract}

Keywords: neutrino physics, flavour physics, beyond standard model

DOI: 10.31526/ACP.BSM-2021.33

\section{INTRODUCTION}

The discovery of neutrino oscillations has established that neutrinos are massive particles and that there is lepton mixing, which must be accounted for in extensions of the Standard Model (SM). From a theoretical perspective, the seesaw mechanism [1, 2, 3, 4 . 5. 6. 7) offers an elegant framework for the explanation of the origin of neutrino masses and lepton mixing.

As opposed to the canonical type-I seesaw, where very heavy "right-handed" (RH) neutrinos or tiny Yukawa couplings are required to generate small neutrino masses, in the inverse seesaw (ISS) [5, 6, 7] neutrino mass suppression is achieved by small lepton-number violating (LNV) parameters. In this case, small Majorana neutrino masses can be generated with RH neutrino masses at the $\mathrm{TeV}$ scale (or below) and $\mathcal{O}(1)$ Yukawa couplings. As a result, the mixing between the (active) light neutrinos and the new (sterile) states can be sizeable for sterile neutrino masses around electroweak scale. The presence of new Majorana fermions interacting with SM leptons and gauge bosons motivates beyond the SM phenomenological studies, making the ISS a perfect theoretical framework to guide new physics probes.

There is a lack of a principle to explain the flavour structure of the SM, i.e., the fermion mass spectra and observed mixing patterns. This flavour puzzle provides a strong motivation for building models with additional particle content and symmetries. One of the simplest approaches is the implementation of texture zeros in the Yukawa couplings and mass matrices imposed by continuous $\mathrm{U}(1)$ and/or discrete $\mathbb{Z}_{N}$ transformations [8. 9]. In the SM extended with $\mathrm{RH}$ neutrinos, the realisation of texture zeros with such symmetries is not compatible with data since they lead to massless charged leptons and/or vanishing lepton mixing angles [9, 10]. To surmount this difficulty we can work in the two-Higgs doublet model (2HDM) [11].

Inspired by the above ideas, we consider the ISS $(2,2)$, which is the minimal setup composed of two RH neutrinos and two sterile singlet fermions [12], within the 2HDM supplemented with Abelian symmetries to ensure maximal predictability, i.e., to impose the most constraining flavour structure, in order to accommodated the charged-lepton masses and neutrino data, as well as, fulfilling all relevant phenomenological constraints, namely the ones on charged lepton flavour-violating (cLFV) processes. This is realised by adding to the SM another scalar doublet and two complex scalar singlets which, after spontaneous symmetry breaking (SSB), generate all relevant mass terms required to implement the ISS(2,2). The work presented here follows closely Ref. [13].

\footnotetext{
\$Speaker
} 


\section{INVERSE SEESAW MECHANISM}

The ISS mechanism is implemented by extending the SM particle content with $n_{R}$ RH neutrinos $v_{R}$ and $n_{S}$ sterile fermion singlets $s$, leading to the $\operatorname{ISS}\left(n_{R}, n_{s}\right)$. Hence, the generic mass Lagrangian for leptons, in the flavour basis, is given by

$$
-\mathcal{L}_{\text {mass }}=\overline{e_{L}} \mathbf{M}_{\ell} e_{R}+\frac{1}{2} \overline{N_{L}^{c}} \mathcal{M} N_{L}+\text { H.c. , } \mathcal{M}=\left(\begin{array}{ccc}
0 & \mathbf{M}_{D}^{*} & 0 \\
\mathbf{M}_{D}^{\dagger} & 0 & \mathbf{M}_{R} \\
0 & \mathbf{M}_{R}^{T} & \mathbf{M}_{s}
\end{array}\right),
$$

where $\mathbf{M}_{\ell}$ is the $3 \times 3$ charged-lepton mass matrix and $N_{L}=\left(v_{L}, v_{R}^{c}, s\right)^{T}$ of dimension $n_{f}=3+n_{R}+n_{s}$ with $v_{L}=\left(v_{e L}, v_{\mu L}, v_{\tau L}\right)^{T}$, $v_{R}=\left(v_{R 1}, \ldots, v_{R n_{R}}\right)^{T}, s=\left(s_{1}, \ldots, s_{n_{s}}\right)^{T}$. For a given fermion field $\psi$ we have $\psi^{c} \equiv C \bar{\psi}^{T}$ with $C$ being the charge conjugation matrix. Furthermore, the full $n_{f} \times n_{f}$ neutrino mass matrix $\mathcal{M}$ is composed by $\mathbf{M}_{D}$ a $3 \times n_{R}$ Dirac-type mass matrix, $\mathbf{M}_{R}$ a $n_{R} \times n_{s}$ matrix, and $\mathbf{M}_{s}$ a LNV $n_{s} \times n_{s}$ Majorana mass matrix. The latter can be naturally small in the 't Hooft [14] sense, since if we set this matrix to zero lepton number conservation is restored.

The charged-lepton mass matrix is bidiagonalised through the unitary transformations $e_{L, R} \rightarrow \mathbf{V}_{L, R} e_{L, R}$

$$
\mathbf{V}_{L}^{\dagger} \mathbf{M}_{\ell} \mathbf{V}_{R}=\mathbf{D}_{\ell}=\operatorname{diag}\left(m_{e}, m_{\mu}, m_{\tau}\right)
$$

with $m_{e, \mu, \tau}$ being the charged-lepton masses. For a given $\mathbf{M}_{\ell}, \mathbf{V}_{L, R}$ are determined by diagonalising the Hermitian matrices $\mathbf{H}_{\ell}=$ $\mathbf{M}_{\ell} \mathbf{M}_{\ell}^{\dagger}$ and $\mathbf{H}_{\ell}^{\prime}=\mathbf{M}_{\ell}^{\dagger} \mathbf{M}_{\ell}$. The weak-basis states $N_{L}$ are related to the mass eigenstates $\left(v_{1}, \ldots, v_{n_{f}}\right)^{T}$ by a $n_{f} \times n_{f}$ unitary matrix $\mathcal{U}$,

$$
N_{L}=\mathcal{U}\left(v_{1}, \ldots, v_{n_{f}}\right)_{L}^{T}
$$

such that $\mathcal{M}$ is diagonalised as

$$
\mathcal{U}^{T} \mathcal{M} \mathcal{U}=\mathcal{D}_{v}=\operatorname{diag}\left(m_{1}, \ldots, m_{n_{f}}\right)
$$

where $m_{1, \ldots, n_{f}}$ are the $n_{f}$ physical Majorana neutrino masses. In the ISS approximation limit $\mathbf{M}_{s}, \mathbf{M}_{D} \ll \mathbf{M}_{R}$, the mass matrix $\mathcal{M}$ of Eq. (1) can be block-diagonalised by writing

$$
\mathcal{M}=\left(\begin{array}{c|cc}
0 & \mathbf{M}_{D}^{*} & 0 \\
\hline \mathbf{M}_{D}^{\dagger} & 0 & \mathbf{M}_{R} \\
0 & \mathbf{M}_{R}^{T} & \mathbf{M}_{s}
\end{array}\right) \equiv\left(\begin{array}{cc}
0 & \mathbf{M}_{D}^{\prime} \\
\mathbf{M}_{D}^{\prime T} & \mathbf{M}_{R}^{\prime}
\end{array}\right)
$$

The full unitary matrix $\mathcal{U}$ of Eq. (4) is parameterised as [15]

$$
\mathcal{U}=\left(\begin{array}{cc}
\sqrt{\mathbb{1}-\mathbf{F F}^{\dagger}} & \mathbf{F} \\
-\mathbf{F}^{+} & \sqrt{\mathbb{1}-\mathbf{F}^{\dagger} \mathbf{F}}
\end{array}\right)\left(\begin{array}{cc}
\mathbf{U}_{v} & 0 \\
0 & \mathbf{U}_{s}
\end{array}\right)
$$

where $\mathbf{F}$ is a $3 \times\left(n_{R}+n_{S}\right)$ matrix given at first order in the seesaw approximation by the following expression

$$
\mathbf{F} \simeq \mathbf{M}_{D}^{* *}\left(\mathbf{M}_{R}^{\prime *}\right)^{-1} \simeq\left(0, \mathbf{M}_{D}\left(\mathbf{M}_{R}^{\dagger}\right)^{-1}\right)
$$

The above block-diagonalisation procedure leads to the $\left(n_{R}+n_{s}\right) \times\left(n_{R}+n_{s}\right)$ mass matrix $\mathbf{M}_{\text {heavy }} \simeq \mathbf{M}_{R}^{\prime}$, diagonalised by $\mathbf{U}_{s}$ and yielding $n_{R}+n_{S}$ heavy neutrinos. Furthermore, we obtain the $3 \times 3$ effective light-neutrino mass matrix

$$
-\mathbf{M}_{\mathrm{eff}}=\mathbf{F}^{*} \mathbf{M}_{R}^{\prime} \mathbf{F}^{\dagger}=\mathbf{M}_{D}^{*}\left(\mathbf{M}_{R} \mathbf{M}_{S}^{-1} \mathbf{M}_{R}^{T}\right)^{-1} \mathbf{M}_{D}^{\dagger}
$$

which is diagonalised through a unitary rotation of the active neutrino fields $v_{L} \rightarrow \mathbf{U}_{v} v_{L}$ leading to

$$
\mathbf{U}_{v}^{T} \mathbf{M}_{\text {eff }} \mathbf{U}_{v}=\mathbf{D}_{v}=\operatorname{diag}\left(\tilde{m}_{1}, \tilde{m}_{2}, \tilde{m}_{3}\right),
$$

with $\tilde{m}_{1,2,3}$ being the physical light neutrino masses in the ISS approximation. From the diagonalisation of the matrix $\mathbf{H}_{\mathrm{eff}}=$ $\mathbf{M}_{\text {eff }} \mathbf{M}_{\text {eff }}^{\dagger}$ we obtain the unitary matrix $\mathbf{U}_{v}$. This yields the unitary lepton mixing matrix

$$
\mathbf{U}^{\prime}=\mathbf{V}_{L}^{\dagger} \mathbf{U}_{v}
$$

For massive Majorana neutrinos $\mathbf{U}^{\prime}$ is parameterised by three mixing angles $\theta_{12}, \theta_{23}$, and $\theta_{13}$, and three CP-violating phases: the Dirac-type phase $\delta$ and two Majorana-type phases $\alpha_{21}$ and $\alpha_{31}$ [16],

$$
\mathbf{U}^{\prime}=\left(\begin{array}{ccc}
c_{12} c_{13} & s_{12} c_{13} & s_{13} \\
-s_{12} c_{23}-c_{12} s_{23} s_{13} e^{i \delta} & c_{12} c_{23}-s_{12} s_{23} s_{13} e^{i \delta} & s_{23} c_{13} e^{i \delta} \\
s_{12} s_{23}-c_{12} c_{23} s_{13} e^{i \delta} & -c_{12} s_{23}-s_{12} c_{23} s_{13} e^{i \delta} & c_{23} c_{13} e^{i \delta}
\end{array}\right)\left(\begin{array}{ccc}
1 & 0 & 0 \\
0 & e^{i \alpha_{21}} & 0 \\
0 & 0 & e^{i \alpha_{31}}
\end{array}\right),
$$




\begin{tabular}{ccc}
\hline Parameter & Best Fit $\pm 1 \sigma$ & $3 \sigma$ range \\
\hline$\theta_{12}\left({ }^{\circ}\right)$ & $34.3 \pm 1.0$ & $31.4 \rightarrow 37.4$ \\
$\theta_{23}\left(^{\circ}\right)[\mathrm{NO}]$ & $48.79_{-1.25}^{+0.93}$ & $41.63 \rightarrow 51.32$ \\
$\theta_{23}\left(^{\circ}\right)[\mathrm{IO}]$ & $48.79_{-1.30}^{+1.04}$ & $41.88 \rightarrow 51.30$ \\
$\theta_{13}\left(^{\circ}\right)[\mathrm{NO}]$ & $8.58_{-0.15}^{+0.11}$ & $8.16 \rightarrow 8.94$ \\
$\theta_{13}\left(^{\circ}\right)[\mathrm{IO}]$ & $8.63_{-0.15}^{+0.11}$ & $8.21 \rightarrow 8.99$ \\
$\delta\left(^{\circ}\right)[\mathrm{NO}]$ & $216_{-25}^{+41}$ & $144 \rightarrow 360$ \\
$\delta\left(^{\circ}\right)[\mathrm{IO}]$ & $277_{-24}^{+23}$ & $205 \rightarrow 342$ \\
$\Delta m_{21}^{2}\left(\times 10^{-5} \mathrm{eV}^{2}\right)$ & $7.50_{-0.20}^{+0.22}$ & $6.94 \rightarrow 8.14$ \\
$\left|\Delta m_{31}^{2}\right|\left(\times 10^{-3} \mathrm{eV}^{2}\right)[\mathrm{NO}]$ & $2.56_{-0.04}^{+0.03}$ & $2.46 \rightarrow 2.65$ \\
$\left|\Delta m_{31}^{2}\right|\left(\times 10^{-3} \mathrm{eV}^{2}\right)[\mathrm{IO}]$ & $2.46 \pm 0.03$ & $2.37 \rightarrow 2.55$ \\
\hline
\end{tabular}

TABLE 1: Current neutrino data obtained by the global fit of three flavour oscillation parameters from Ref. [18].

where we use the notation $c_{i j} \equiv \cos \theta_{i j}$ and $s_{i j} \equiv \sin \theta_{i j}$. In Table 1 are presented the results from the most recent global fit of neutrino oscillation parameters [18]. Note that $\Delta m_{21}^{2}=m_{2}^{2}-m_{1}^{2}, \Delta m_{31}^{2}=m_{3}^{2}-m_{1}^{2}$. Both mass orderings are considered namely, normal ordering (NO) $\left(m_{1}<m_{2}<m_{3}\right)$ and inverted ordering (IO) $\left(m_{3}<m_{1}<m_{2}\right)$. The lepton sector has a total of twelve parameters: three charged lepton masses, three light neutrino masses, three mixing angles and three phases. In the massless neutrino case there is only one physical Majorana phase, thus, the total number of physical parameters in the lepton sector is reduced to ten.

Consider the rectangular $3 \times n_{f}$ matrix $\mathbf{W}_{\alpha j} \equiv \mathcal{U}_{\alpha j}\left(\alpha=e, \mu, \tau, j=1, \ldots, n_{f}\right)$ which, according to Eq. 6), can be decomposed in the form

$$
\mathbf{W}=\left(\sqrt{\mathbb{1}-\mathbf{F F}^{\dagger}} \mathbf{U}_{v}, \mathbf{F U}_{s}\right) \equiv\left(\mathbf{W}_{v}, \mathbf{W}_{s}\right),
$$

with $\mathbf{W}_{v}$ and $\mathbf{W}_{s}$ being $3 \times 3$ and $3 \times\left(n_{R}+n_{s}\right)$ matrices, respectively. The presence of additional fermionic states leads to activeneutrino mixing determined by the non-unitary matrix

$$
\mathbf{U}=\mathbf{V}_{L}^{\dagger} \mathbf{W}_{v}=(\mathbb{1}-\boldsymbol{\eta}) \mathbf{U}^{\prime},
$$

where $\mathbf{U}^{\prime}$ is the unitary lepton mixing matrix given by Eq. 10 and $\boldsymbol{\eta}$ is an Hermitian matrix that encodes deviations from unitarity of $\mathbf{U}$. Furthermore, $\mathbf{V}_{L}^{\dagger} \mathbf{W}_{s}$ defines the mixing between the three active neutrinos and the $n_{R}+n_{s}$ sterile states in the physical charged-lepton basis, which at first order in $\mathbf{F}$ is

$$
\mathbf{V}_{L}^{\dagger} \mathbf{W}_{s}=\mathbf{V}_{L}^{\dagger} \mathbf{F} \mathbf{U}_{s} \simeq \mathbf{V}_{L}^{\dagger}\left(0, \mathbf{M}_{D}\left(\mathbf{M}_{R}^{\dagger}\right)^{-1}\right) \mathbf{U}_{s}
$$

The parameters $\eta_{\alpha \beta}$ encoding deviations from unitarity can be expressed solely in terms of the active-sterile mixing through the relations

$$
\boldsymbol{\eta}_{\alpha \beta}=\frac{1}{2} \sum_{j=4}^{n_{f}} \mathbf{B}_{\alpha j} \mathbf{B}_{\beta j}^{*}, \mathbf{B}_{\alpha j}=\sum_{k=1}^{3}\left(\mathbf{V}_{L}^{*}\right)_{k \alpha} \mathbf{W}_{k j} .
$$

The mixing between the light and sterile neutrinos is also given by the matrix elements $\mathbf{B}_{\alpha j}$ for $\alpha=e, \mu, \tau$ and $j=4, \ldots, n_{f}$.

\section{MAXIMALLY-RESTRICTIVE TEXTURES FOR LEPTONS}

In this section, we identify the maximally-restrictive textures for the set of matrices $\left(\mathbf{M}_{\ell}, \mathbf{M}_{D}, \mathbf{M}_{R}, \mathbf{M}_{s}\right)$ compatible with neutrino oscillation data within the minimal ISS $(2,2)$ framework, where $n_{R}=n_{S}=2$ and $n_{f}=7$. For the texture-zero analysis we assuming the seesaw approximation given in Eq. (8). To identify the compatible textures we follow a standard $\chi^{2}$-analysis using the function

$$
\chi^{2}(x)=\sum_{i} \frac{\left[\mathcal{P}_{i}(x)-\mathcal{O}_{i}\right]^{2}}{\sigma_{i}^{2}},
$$

with $x$ denoting the input parameters, i.e., the matrix elements of $\mathbf{M}_{\ell}, \mathbf{M}_{D}, \mathbf{M}_{R}$ and $\mathbf{M}_{s} ; \mathcal{P}_{i}(x)$ is the model prediction for a given observable with best-fit value $\mathcal{O}_{i}$; and $\sigma_{i}$ denotes its $1 \sigma$ experimental uncertainty. In our search for viable sets $\left(\mathbf{M}_{\ell}, \mathbf{M}_{D}, \mathbf{M}_{R}, \mathbf{M}_{s}\right)$, we require the charged-lepton masses to be at their central values [17], such that the $\chi^{2}$-function is minimised only with respect to the six remaining neutrino oscillation observables, using the data reported in Table 1] [18]. Note that, in the ISS(2,2) framework, there will always be a massless neutrino $\left(\tilde{m}_{1}=0\right.$ for $\mathrm{NO}$ or $\tilde{m}_{3}=0$ for $\left.\mathrm{IO}\right)$. 


\begin{tabular}{ccc}
\hline \multicolumn{3}{c}{$\mathbf{M}_{\ell}=6^{\ell}$} \\
\hline $\mathbf{M}_{D}$ & $\mathbf{M}_{R}$ & $\mathbf{M}_{5}$ \\
\hline $\mathrm{T}_{1}$ & $\mathrm{~T}_{14}$ & $\mathrm{~T}_{23}$ \\
$\mathrm{~T}_{4}$ & $\mathrm{~T}_{14}$ & $\mathrm{~T}_{23}$ \\
$\mathrm{~T}_{5}$ & $\mathrm{~T}_{14}$ & $\mathrm{~T}_{23}$ \\
$\mathrm{~T}_{14}$ & $\mathrm{~T}_{1}$ & $\mathrm{~T}_{23}$ \\
$\mathrm{~T}_{16}$ & $\mathrm{~T}_{1}$ & $\mathrm{~T}_{23}$ \\
$\mathrm{~T}_{23}$ & $\mathrm{~T}_{1}$ & $\mathrm{~T}_{23}$ \\
$\mathrm{~T}_{25}$ & $\mathrm{~T}_{1}$ & $\mathrm{~T}_{23}$ \\
$\mathrm{~T}_{36}$ & $\mathrm{~T}_{1}$ & $\mathrm{~T}_{23}$ \\
$\mathrm{~T}_{45}$ & $\mathrm{~T}_{1}$ & $\mathrm{~T}_{23}$ \\
\hline
\end{tabular}

\begin{tabular}{ccc}
\hline \multicolumn{3}{c}{$\mathbf{M}_{\ell}=5_{1}^{\ell}$} \\
\hline $\mathbf{M}_{D}$ & $\mathbf{M}_{R}$ & $\mathbf{M}_{s}$ \\
\hline $\mathrm{T}_{13}$ & $\mathrm{~T}_{14}$ & $\mathrm{~T}_{23}$ \\
$\mathrm{~T}_{14}$ & $\mathrm{~T}_{14}$ & $\mathrm{~T}_{23}$ \\
$\mathrm{~T}_{16}$ & $\mathrm{~T}_{14}$ & $\mathrm{~T}_{23}$ \\
$\mathrm{~T}_{35}$ & $\mathrm{~T}_{14}$ & $\mathrm{~T}_{23}$ \\
$\mathrm{~T}_{45}$ & $\mathrm{~T}_{14}$ & $\mathrm{~T}_{23}$ \\
\hline
\end{tabular}

\begin{tabular}{ccc}
\hline \multicolumn{3}{c}{$\mathbf{M}_{\ell}=4_{1,2,3}^{\ell}$} \\
\hline $\mathbf{M}_{D}$ & $\mathbf{M}_{R}$ & $\mathbf{M}_{s}$ \\
\hline $\mathrm{T}_{124}$ & $\mathrm{~T}_{14}$ & $\mathrm{~T}_{23}$ \\
$\mathrm{~T}_{125}$ & $\mathrm{~T}_{14}$ & $\mathrm{~T}_{23}$ \\
$\mathrm{~T}_{134}$ & $\mathrm{~T}_{14}$ & $\mathrm{~T}_{23}$ \\
$\mathrm{~T}_{136}$ & $\mathrm{~T}_{14}$ & $\mathrm{~T}_{23}$ \\
$\mathrm{~T}_{145}$ & $\mathrm{~T}_{14}$ & $\mathrm{~T}_{23}$ \\
$\mathrm{~T}_{146}$ & $\mathrm{~T}_{14}$ & $\mathrm{~T}_{23}$ \\
$\mathrm{~T}_{156}$ & $\mathrm{~T}_{14}$ & $\mathrm{~T}_{23}$ \\
$\mathrm{~T}_{345}$ & $\mathrm{~T}_{14}$ & $\mathrm{~T}_{23}$ \\
$\mathrm{~T}_{456}$ & $\mathrm{~T}_{14}$ & $\mathrm{~T}_{23}$ \\
\hline
\end{tabular}

TABLE 2: Maximally-restrictive texture sets with $\mathbf{M}_{\ell}=6^{\ell}$ (left), $5_{1}^{\ell}$ (centre) and $4_{1,2,3}^{\ell}$ (right).

$$
\begin{array}{rlrl}
4_{1}^{\ell} \sim\left(\begin{array}{ccc}
0 & 0 & \times \\
0 & \times & 0 \\
\times & \times & \times
\end{array}\right) & 4_{2}^{\ell} \sim\left(\begin{array}{ccc}
0 & 0 & \times \\
0 & \times & \times \\
\times & 0 & \times
\end{array}\right) & 4_{3}^{\ell} \sim\left(\begin{array}{ccc}
0 & 0 & \times \\
0 & \times & \times \\
\times & \times & 0
\end{array}\right) \\
5_{1}^{\ell} \sim\left(\begin{array}{ccc}
0 & 0 & \times \\
0 & \times & 0 \\
\times & 0 & \times
\end{array}\right) & 6^{\ell} \sim\left(\begin{array}{ccc}
\times & 0 & 0 \\
0 & \times & 0 \\
0 & 0 & \times
\end{array}\right)
\end{array}
$$

TABLE 3: Charged-lepton mass matrix $\mathbf{M}_{\ell}$ textures.

We consider compatibility with data, for a given set of input matrices, if the deviation of each neutrino observable from its experimental value is at most $3 \sigma$ at the $\chi^{2}$-minimum [8, 9. If this is verified, we test the compatibility of the textures at $1 \sigma$. We shall use the following sequential notation to label the position of the matrix elements of a given $3 \times 2$ and $2 \times 2$ texture T, respectively,

$$
\left(\begin{array}{ll}
1 & 2 \\
3 & 4 \\
5 & 6
\end{array}\right),\left(\begin{array}{ll}
1 & 2 \\
3 & 4
\end{array}\right),
$$

where the position of any vanishing element labelled $i$ is denoted with a subscript, i.e., $\mathrm{T}_{i}$.

In Table 2 2 we present the maximally-restrictive texture zero sets $\left(\mathbf{M}_{\ell}, \mathbf{M}_{D}, \mathbf{M}_{R}, \mathbf{M}_{s}\right)$ that are compatible with oscillation data for NO. Note that, these sets of matrices are also viable for IO. Additionally, all the sets are compatible with data at $1 \sigma$. The labelling used for the $\mathbf{M}_{\ell}$ matrix in Table 3 follows Ref. [19].

\section{ABELIAN SYMMETRY REALISATION OF COMPATIBLE TEXTURES}

As mentioned before, our minimal setup will require at least two Higgs doublets $\Phi_{a}(a=1,2)$. Furthermore, to avoid bare mass terms in the Lagrangian, we add two complex scalar fields $S_{a}(a=1,2)$, such that $\mathbf{M}_{s}$ and $\mathbf{M}_{R}$ are dynamically generated through couplings of $S_{1}$ and $S_{2}$ with $\overline{s^{c}} s$ and $\overline{v_{R}} s$, respectively. Hence, the Yukawa Lagrangian relevant for our work is

$$
-\mathcal{L}_{\text {Yuk. }}=\overline{\ell_{L}} \mathbf{Y}_{\ell}^{a} \Phi_{a} e_{R}+\overline{\ell_{L}} \mathbf{Y}_{D}^{a} \tilde{\Phi}_{a} v_{R}+\frac{1}{2} \overline{s^{c}}\left(\mathbf{Y}_{s}^{1} S_{1}+\mathbf{Y}_{s}^{2} S_{1}^{*}\right) s+\overline{v_{R}}\left(\mathbf{Y}_{R}^{1} S_{2}+\mathbf{Y}_{R}^{2} S_{2}^{*}\right) s+\text { H.c., }
$$

where the sum over $a$ is implicit. Upon SSB, the scalar fields acquire non-zero vacuum expectation values (VEV)

$$
\left\langle\phi_{1}^{0}\right\rangle=v \cos \beta,\left\langle\phi_{2}^{0}\right\rangle=v \sin \beta, \tan \beta=\frac{v_{2}}{v_{1}},\left\langle S_{1}\right\rangle=u_{1} e^{i \xi},\left\langle S_{2}\right\rangle=u_{2},
$$

and the above Yukawa Lagrangian yields the generic mass Lagrangian of Eq. (1) for the ISS $(2,2)$.

To identify which of the maximally-restrictive texture sets compatible with neutrino data, presented in Section 3 can be realised by imposing discrete or continuous Abelian symmetries, we apply two complementary methods: the canonical [20] and Smith normal form (SNF) [21] methods. We closely follow the methodology employed in Refs. [8, 9].

In Table 4 we present the realisable mass matrix textures and their corresponding Yukawa decompositions. Table 5 displays, the Abelian symmetry group that realises the texture set and the associated transformation charges for each field. For all cases, the full texture decomposition is imposed by the $\mathrm{U}(1)_{\mathrm{F}}$ symmetry alone. The $\mathrm{U}(1)$ symmetry does not impose any texture zero on the mass matrices, but restricts the Yukawa Lagrangian to the form given in Eq. [18, where the term with $\mathbf{Y}_{R}^{2}$ is forbidden and $\mathbf{Y}_{R} \equiv \mathbf{Y}_{R}^{1}$. Since for all realisable cases $\mathbf{M}_{R}$ and $\mathbf{M}_{s}$ are fixed by the textures $\mathrm{T}_{14}$ and $\mathrm{T}_{23}$, respectively, from now on, we refer to each case through the pair notation $\left(\mathbf{M}_{\ell}, \mathbf{M}_{D}\right)$. 


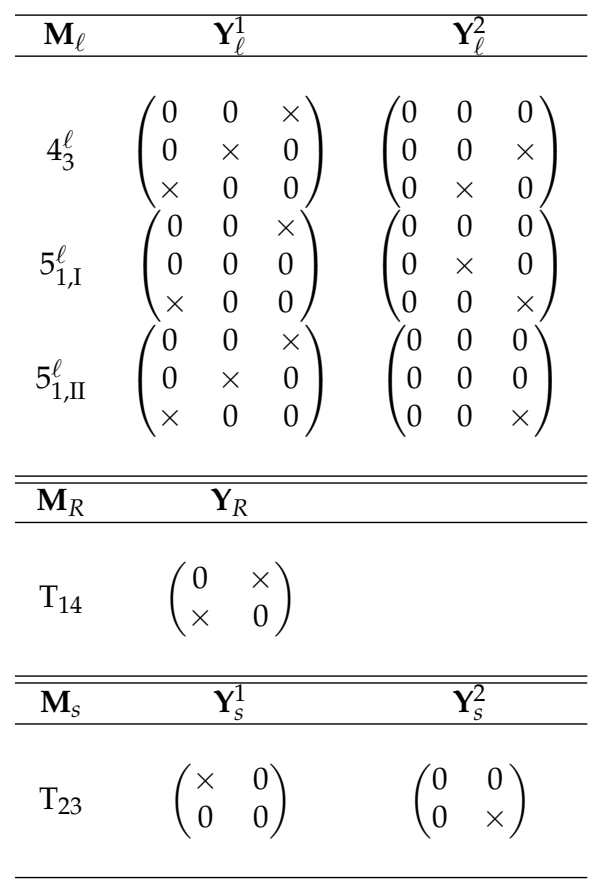

\begin{tabular}{ccc}
\hline $\mathbf{M}_{D}$ & $\mathbf{Y}_{D}^{1}$ & $\mathbf{Y}_{D}^{2}$ \\
$\mathrm{~T}_{45}$ & $\left(\begin{array}{cc}\times & 0 \\
0 & 0 \\
0 & \times\end{array}\right)$ & $\left(\begin{array}{ll}0 & \times \\
\times & 0 \\
0 & 0\end{array}\right)$ \\
$\mathrm{T}_{124}$ & $\left(\begin{array}{ll}0 & 0 \\
0 & 0 \\
\times & 0\end{array}\right)$ & $\left(\begin{array}{ll}0 & 0 \\
\times & 0 \\
0 & \times\end{array}\right)$ \\
$\mathrm{T}_{456}$ & $\left(\begin{array}{ll}0 & \times \\
\times & 0 \\
0 & 0\end{array}\right)$ & $\left(\begin{array}{ll}\times & 0 \\
0 & 0 \\
0 & 0\end{array}\right)$ \\
$\mathrm{T}_{136, \mathrm{I}}$ & $\left(\begin{array}{ll}0 & 0 \\
0 & \times \\
0 & 0\end{array}\right)$ & $\left(\begin{array}{ll}0 & \times \\
0 & 0 \\
\times & 0\end{array}\right)$ \\
$\mathrm{T}_{136, \mathrm{II}}$ & $\left(\begin{array}{ll}0 & \times \\
0 & 0 \\
0 & 0\end{array}\right)$ & $\left(\begin{array}{ll}0 & 0 \\
0 & \times \\
\times & 0\end{array}\right)$ \\
$\mathrm{T}_{146, \mathrm{I}}$ & $\left(\begin{array}{ll}0 & \times \\
0 & 0 \\
\times & 0\end{array}\right)$ & $\left(\begin{array}{ll}0 & 0 \\
\times & 0 \\
0 & 0\end{array}\right)$ \\
$\mathrm{T}_{146, \mathrm{II}}$ & $\left(\begin{array}{cc}0 & \times \\
\times & 0 \\
0 & 0\end{array}\right)$ & $\left(\begin{array}{ll}0 & 0 \\
0 & 0 \\
\times & 0\end{array}\right)$
\end{tabular}

TABLE 4: Decomposition of mass matrices into the Yukawa textures following Eq. 18 .

\begin{tabular}{ccccccc}
\hline & & $\left(5_{1, \mathrm{I}}^{\ell}, \mathrm{T}_{45}\right)$ & $\left(4_{3}^{\ell}, \mathrm{T}_{124}\right)$ & $\left(4_{3}^{\ell}, \mathrm{T}_{456}\right)$ & $\left(4_{3}^{\ell}, \mathrm{T}_{136, \mathrm{I}}\right)$ & $\left(4_{3}^{\ell}, \mathrm{T}_{146, \mathrm{I}}\right)$ \\
Fields & $\mathrm{U}(1)$ & $\mathbb{Z}_{2} \times \mathrm{U}(1)_{\mathrm{F}}$ & $\mathbb{Z}_{2} \times \mathrm{U}(1)_{\mathrm{F}}$ & $\mathbb{Z}_{2} \times \mathrm{U}(1)_{\mathrm{F}}$ & $\mathbb{Z}_{4} \times \mathrm{U}(1)_{\mathrm{F}}$ & $\mathbb{Z}_{4} \times \mathrm{U}(1)_{\mathrm{F}}$ \\
\hline$\Phi_{1}$ & 0 & $(1,1)$ & $(0,-5)$ & $(1,1)$ & $(1,2)$ & $(0,1)$ \\
$\Phi_{2}$ & 0 & $(0,-1)$ & $(1,-3)$ & $(0,-1)$ & $(0,1)$ & $(3,0)$ \\
$S_{1}$ & 0 & $(0,2)$ & $(0,-2)$ & $(0,-2)$ & $(0,-2)$ & $(0,-2)$ \\
$S_{2}$ & 1 & $(0,0)$ & $(0,0)$ & $(1,0)$ & $(0,0)$ & $(0,0)$ \\
$\ell_{e_{L}}$ & 1 & $(1,0)$ & $(0,0)$ & $(0,0)$ & $(2,0)$ & $(2,0)$ \\
$\ell_{\mu_{L}}$ & 1 & $(0,2)$ & $(1,2)$ & $(1,-2)$ & $(1,-1)$ & $(1,-1)$ \\
$\ell_{\tau_{L}}$ & 1 & $(0,-2)$ & $(0,4)$ & $(0,-4)$ & $(0,-2)$ & $(0,-2)$ \\
$e_{R}$ & 1 & $(1,-3)$ & $(0,9)$ & $(1,-5)$ & $(3,-4)$ & $(0,-3)$ \\
$\mu_{R}$ & 1 & $(0,3)$ & $(1,7)$ & $(0,-3)$ & $(0,-3)$ & $(1,-2)$ \\
$\tau_{R}$ & 1 & $(0,-1)$ & $(0,5)$ & $(1,-1)$ & $(1,-2)$ & $(2,-1)$ \\
$v_{R_{1}}$ & 1 & $(0,1)$ & $(0,-1)$ & $(0,-1)$ & $(0,-1)$ & $(0,-1)$ \\
$v_{R_{2}}$ & 1 & $(1,-1)$ & $(1,1)$ & $(1,1)$ & $(2,1)$ & $(2,1)$ \\
$s_{1}$ & 0 & $(1,-1)$ & $(1,1)$ & $(0,1)$ & $(2,1)$ & $(2,1)$ \\
$s_{2}$ & 0 & $(0,1)$ & $(0,-1)$ & $(1,-1)$ & $(0,-1)$ & $(0,-1)$ \\
\hline
\end{tabular}

TABLE 5: Maximally-restrictive texture sets realisable by an Abelian symmetry group. For a given texture pair we indicate the $\mathbb{Z}_{n}$ charges $q_{n}$ such that the transformation phases are $e^{2 \pi i q_{n} / n}$. The $\mathrm{U}(1)$ and $\mathrm{U}(1)_{\mathrm{F}}$ charges are multiples of the charges $q_{1}$ and $q_{\mathrm{F}}$, respectively.

\section{LEPTON MASSES, MIXING AND LEPTONIC CPV}

Throughout the rest of this work we restrict our phenomenological analysis to the combination $\left(5_{1, \mathrm{I}}^{\ell}, \mathrm{T}_{45}\right)$. We consider the scenario in which CP is imposed at the Lagrangian level. It can be shown that the scalar potential of the fields $\Phi$ and $S_{1,2}$, with specific soft breaking of the $\mathrm{U}(1) \times \mathbb{Z}_{2} \times \mathrm{U}(1)_{\mathrm{F}}$ symmetry, allows for spontaneous CP violation (SCPV) stemming from the complex phase $e^{i \xi}$ of the VEV of the singlet $S_{1}$ (see Ref. [13] for details). The mass matrices are parameterised as

$$
\mathbf{M}_{\ell}=\left(\begin{array}{ccc}
0 & 0 & a_{1} \\
0 & m_{\ell_{1}}^{2} & 0 \\
a_{2} & 0 & a_{4}
\end{array}\right), \mathbf{M}_{D}=\left(\begin{array}{cc}
m_{D_{1}} & m_{D_{3}} \\
m_{D_{4}} & 0 \\
0 & m_{D_{2}}
\end{array}\right), \mathbf{M}_{R}=\left(\begin{array}{cc}
0 & M \\
q M & 0
\end{array}\right), \mathbf{M}_{s}=\left(\begin{array}{cc}
p \mu_{s} e^{i \xi} & 0 \\
0 & \mu_{s} e^{-i \xi}
\end{array}\right),
$$

where all parameters are real. Note that, the charged-lepton state $\ell_{1}$ is decoupled from the remaining ones, leading to three distinct cases of $5_{1}^{\ell_{1}}$ textures with $\ell_{1}=e, \mu, \tau$, labelled as $5_{1}^{e, \mu, \tau}$. The diagonalisation of the charged-lepton mass matrix is performed by the unitary rotations $\mathbf{V}_{L, R}$ with angles $\theta_{L, R}$. Furthermore, $p$ and $q$ are dimensionless rescalings. 

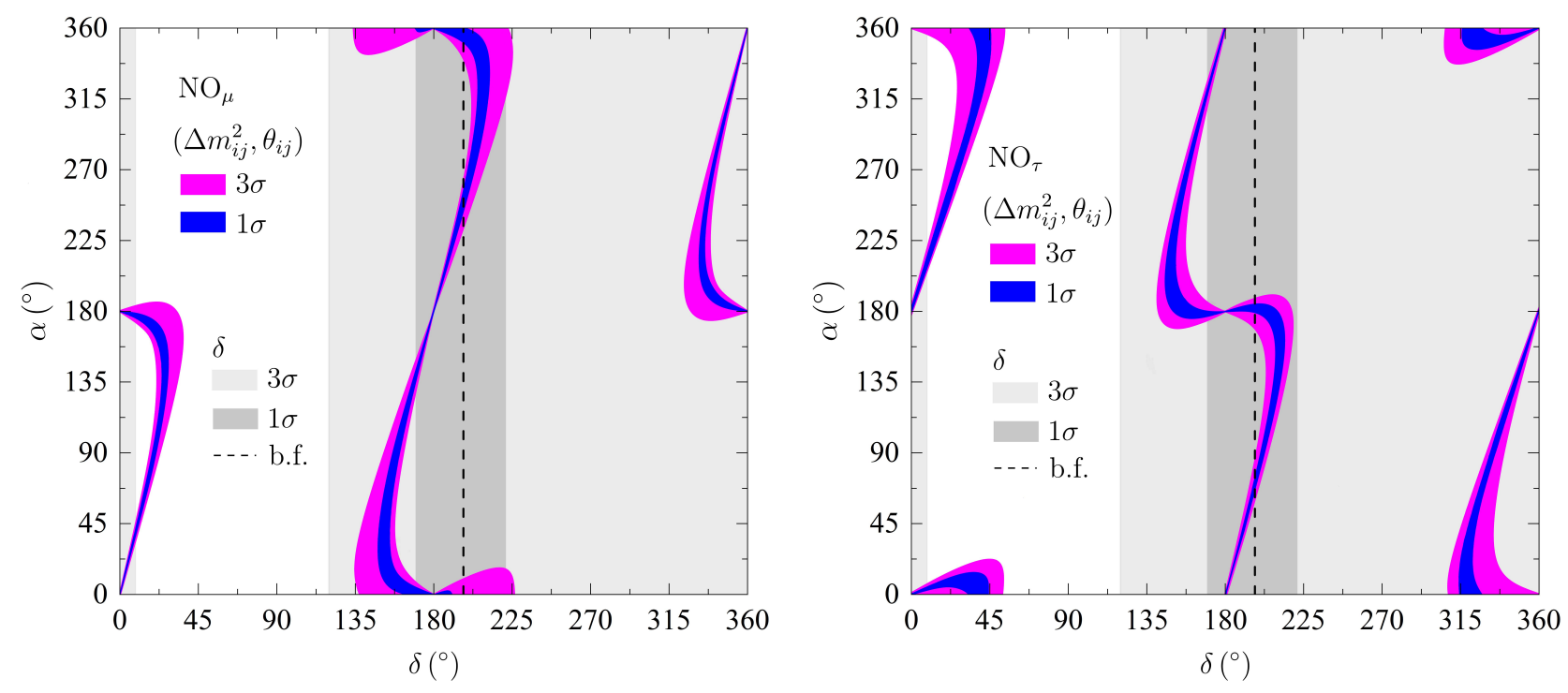

FIGURE 1: Predictions for the Majorana phase $\alpha$ as a function of the Dirac phase $\delta$ varying the neutrino mixing angles $\theta_{i j}$ and masssquared differences $\Delta m_{21}^{2}$ and $\Delta m_{31}^{2}$ in the $1 \sigma$ (blue) and $3 \sigma$ (magenta) ranges given by Table 1 The dark (light) grey vertical band display the $1 \sigma(3 \sigma)$ range for $\delta$ shown in the same table, while the vertical dashed line is the $\delta$ phase best-fit value. The left (right) panel corresponds to the $\mathrm{NO}_{\mu}\left(\mathrm{NO}_{\tau}\right)$ case.

The effective mass matrix $\mathbf{M}_{\text {eff }}$ can be written in terms of six relevant parameters namely the phase $\xi$, angle $\theta_{L}$ and

$$
x=\mu_{s} \frac{m_{D_{4}}^{2}}{M^{2}}, y=\mu_{s} \frac{m_{D_{1}} m_{D_{4}}}{M^{2}}, z=\mu_{s} \frac{m_{D_{2}} m_{D_{3}}}{M^{2}} \frac{p}{q^{2}}, w=\mu_{s} \frac{m_{D_{2}}^{2}}{M^{2}} \frac{p}{q^{2}} .
$$

The matrix $M$ written in terms of the low-energy parameters according to Eqs. 9]-(11) is given by

$$
\mathrm{NO}: M_{i j}=\left[\mathbf{U}^{\prime *} \operatorname{diag}\left(0, \sqrt{\Delta m_{21}^{2}}, \sqrt{\Delta m_{31}^{2}}\right) \mathbf{U}^{\prime \dagger}\right]_{i j}, \mathrm{IO}: M_{i j}=\left[\mathbf{U}^{\prime *} \operatorname{diag}\left(\sqrt{\Delta m_{31}^{2}}, \sqrt{\Delta m_{21}^{2}+\Delta m_{31}^{2}}, 0\right) \mathbf{U}^{\prime \dagger}\right]_{i j},
$$

for both NO and IO neutrino masses. The lepton mixing matrix $\mathbf{U}^{\prime}$ is parametrised as in Eq. (11). The six parameters defining $\mathbf{M}_{\mathrm{eff}}$ are to be compared with the seven low-energy physical parameters, namely three mixing angles $\theta_{i j}$, two neutrino masses and two CPV phases, i.e., the Dirac and Majorana phases, $\delta$ and $\alpha$ defining $M$. This implies a relation between the elements of the effective neutrino mass matrix, which results in a correlation between two low-energy parameters. It can be shown that the said relation for the $5_{1}^{e}$ case is given by

$$
5_{1}^{e}: \arg \left[M_{11}^{* 2} M_{13}^{2} \frac{\mathrm{D}_{12}}{\mathrm{D}_{23}}\right]=0, \mathrm{D}_{i j}=M_{i i} M_{j j}-M_{i j}^{2},
$$

while the corresponding ones for the $5_{1}^{\mu}$ and $5_{1}^{\tau}$ cases are obtained by performing replacements $(11 \rightarrow 12,13 \rightarrow 23)$ and $(11 \rightarrow$ $13,13 \rightarrow 33)$, respectively. For a given set of $\theta_{i j}$ and $\Delta m_{21,31}^{2}$ values, the equations above establish how the CP-phases $\alpha$ and $\delta$ are correlated. Moreover, $\theta_{L}, \xi$ and all parameters in Eq. 21] can be expressed in terms of low-energy neutrino observables.

To establish numerically how $\delta$ and $\alpha$ are related, we vary the mixing angles $\theta_{i j}$ and neutrino mass-squared differences $\Delta m_{21,31}^{2}$ within their experimental $1 \sigma$ and $3 \sigma$ ranges, indicated in Table 1 . while changing $\delta$ from 0 to $2 \pi$. Next, for both NO and IO cases, we compute $M_{i j}$ through Eq. 22]. The Majorana phase $\alpha$ is obtained by solving Eq. 23] for $5_{1}^{e, \mu, \tau}$, leading to the results displayed in Fig. 1. where we specifically present the most interesting cases: $\mathrm{NO}_{\mu}$ and $\mathrm{NO}_{\tau}$ (we use the notation $\mathrm{NO}_{e, \mu, \tau}\left(\mathrm{IO}_{e, \mu, \tau}\right)$ corresponding to $5_{1}^{c, \mu, \tau}$ with a NO (IO) neutrino mass spectrum). The blue (magenta) regions are obtained by taking the $1 \sigma$ ( $3 \sigma$ ) intervals for $\theta_{i j}$ and $\Delta m_{21,31}^{2}$, while the vertical dark (light) grey band marks the current allowed region for the Dirac CP phase $\delta$ at $1 \sigma(3 \sigma)$. The results show a strong correlation between $\alpha$ and $\delta$. The plots exhibit an approximate symmetry under the shift $\delta \rightarrow \delta+\pi$, due to the fact that Eq. 23. is nearly invariant under that transformation at leading order in the smallest mixing angle $\theta_{13}$. The absence of Dirac-type CP violation $(\delta=0, \pi)$ implies $\alpha=k \pi(k \in \mathbb{Z})$. This is confirmed analytically by evaluating the Dirac and Majorana $\mathrm{CP}$ weak basis invariants, $\mathcal{J}_{\text {Dirac }}^{\mathrm{CP}}$ and $\mathcal{J}_{\text {Maj }}^{\mathrm{CP}}[22]$, which turn out to be both proportional to $\sin (2 \xi)$. A future measurement of $\delta$ in the intervals $\left[45^{\circ}, 135^{\circ}\right]$ and $\left[225^{\circ}, 315^{\circ}\right]$ would exclude the cases presented in the figure since, in these ranges, Eq. 23. has no solution.

The seesaw approximation provides analytical insight on the heavy-light mixing properties. We start by diagonalising the effective heavy neutrino mass matrix $\mathbf{M}_{\text {heavy }}$ and we obtain the $4 \times 4$ unitary matrix $\mathbf{U}_{s}$ composed of the angles $\varphi_{1} \simeq \varphi_{2} \simeq \pi / 4$. This leads to two pairs of pseudo-Dirac neutrinos with masses $\tilde{m}_{4,5} \simeq M \mp \mu_{s} / 2$ and $\tilde{m}_{6,7} \simeq q M \mp p \mu_{s} / 2$. The mass differences 

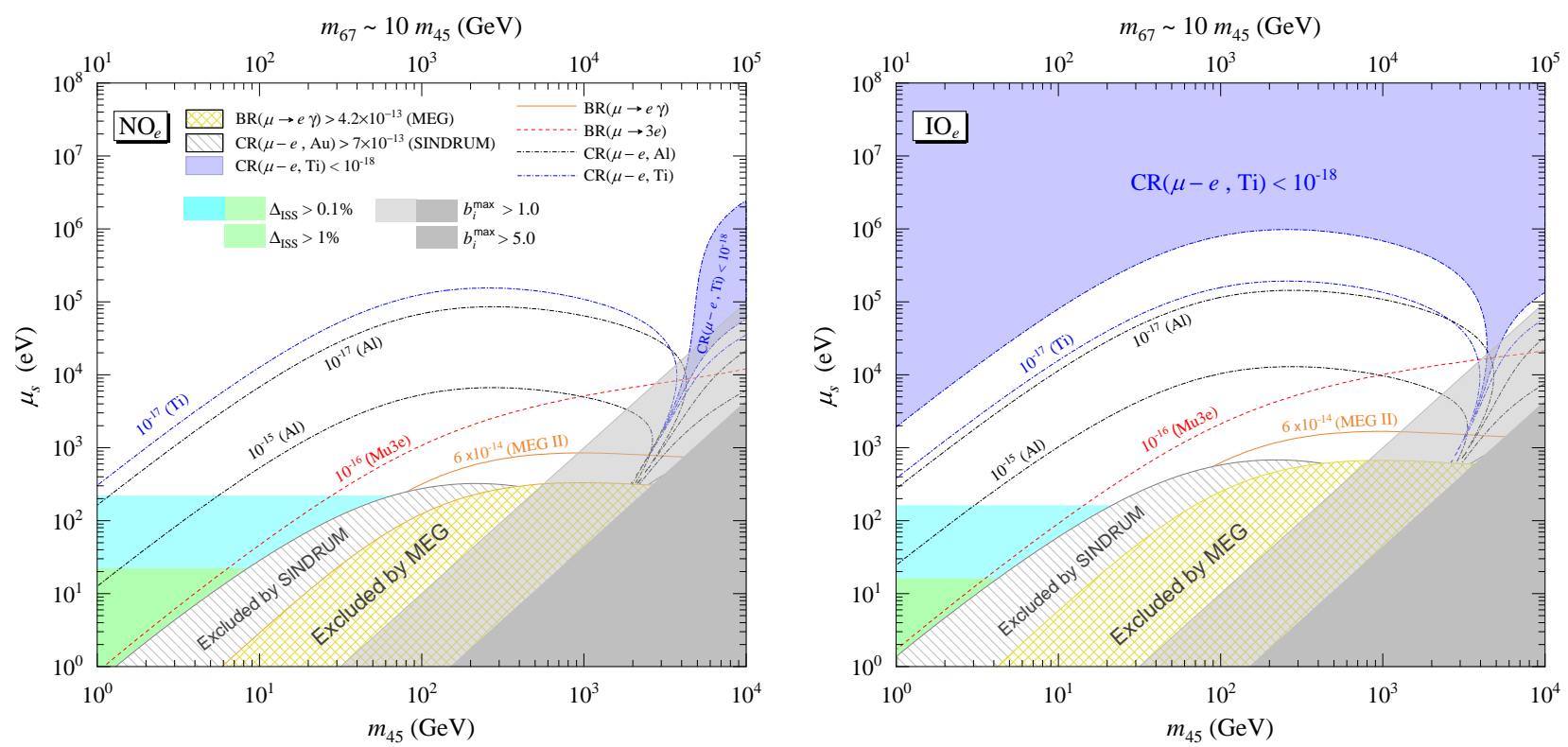

FIGURE 2: Constraints imposed on the $\left(m_{45}, \mu_{s}\right)$ parameter space by the bounds from MEG on BR $(\mu \rightarrow e \gamma)$ (yellow crosshatched region) and SINDRUM II on $\mathrm{CR}(\mu-e, \mathrm{Au})$ (grey hatched region). Are also given the contours corresponding to the future sensitivities projected by the MEG II (solid orange) and Mu3e (red dashed) experiments. The black and blue dash-dotted lines show the contours of $\mathrm{CR}(\mu-e, \mathrm{Al})$ and $\mathrm{CR}(\mu-e, \mathrm{Ti})$, respectively, for values within the projected sensitivities of future experiments (see text for details). Inside the blue shaded region $\mathrm{CR}(\mu-e, \mathrm{Ti})<10^{-18}$. Limits on $b_{i}^{\max }$ and $\Delta_{\mathrm{ISS}}$ are also presented (grey, green and cyan shaded regions). The results are shown for the $5_{1}^{e}$ case with NO (left panel) and IO (right panel).

$\tilde{m}_{5}-\tilde{m}_{4}=\mu_{s}$ and $\tilde{m}_{7}-\tilde{m}_{6}=p \mu_{s}$ are controlled by the small LNV parameter $\mu_{s}$. From the matrix $\mathbf{U}_{s}$ and Eq. (14) we obtain the heavy-light neutrino mixing defined in terms of the matrix B in Eq. 15. Due to the Abelian symmetries imposed in order to realise the maximally-restricted textures, the $\mathbf{B}_{\alpha j}$ for distinct lepton flavours are related to each other by low-energy neutrino parameters. Indeed, for the $5_{1}^{e}$ case we have

$$
\frac{\mathbf{B}_{e 4}}{\mathbf{B}_{\mu 4}} \simeq \frac{\mathbf{B}_{e 5}}{\mathbf{B}_{\mu 5}} \simeq \frac{x}{y c_{L}}, \quad \frac{\mathbf{B}_{\tau 4}}{\mathbf{B}_{\mu 4}} \simeq \frac{\mathbf{B}_{\tau 5}}{\mathbf{B}_{\mu 5}} \simeq \tan \theta_{L}, \frac{\mathbf{B}_{\mu 6}}{\mathbf{B}_{\tau 6}} \simeq \frac{\mathbf{B}_{\mu 7}}{\mathbf{B}_{\tau 7}} \simeq \frac{z-w \tan \theta_{L}}{w+z \tan \theta_{L}}, \quad \mathbf{B}_{e 6} \simeq \mathbf{B}_{e 7} \simeq 0,
$$

where all the above parameters depend solely on the neutrino observables. The corresponding relations for the $5_{1}^{\mu}$ and $5_{1}^{\tau}$ textures are obtained by performing $(e \leftrightarrow \mu)$ and $(e \leftrightarrow \tau)$, respectively.

\section{CHARGED LEPTON FLAVOUR VIOLATION}

Since the ISS model is a paradigm for low-scale neutrino mass generation, it provides a natural scenario for the observation of flavour transitions besides neutrino oscillations. In our framework, the symmetries discussed in Section 4 lay the ground for a testable scenario in light of present and future experimental probes on lepton flavour violating (LFV) processes which we analyse in this section.

In our numerical analysis, we are interested in comparing the tree-level light neutrino parameters obtained from the seesawapproximated $\mathbf{M}_{\text {eff }}$ of Eq. (8) with those from the full neutrino mass matrix $\mathcal{M}$ in Eq. (1). In order to quantify the effect of considering the ISS approximation at lowest order we define

$$
\Delta_{\mathrm{ISS}} \equiv \frac{\left|\Delta m_{31}^{2}-\Delta \tilde{m}_{31}^{2}\right|}{\Delta \tilde{m}_{31}^{2}}, \Delta \tilde{m}_{31}^{2}=\tilde{m}_{3}^{2}-\tilde{m}_{1}^{2}
$$

with the light-neutrino masses $m_{i}$ and $\tilde{m}_{i}$ being determined through Eqs. (4) and (9), respectively.

For numerical computations in the $5_{1}^{e, \mu \tau}$ cases discussed in previous sections, we consider a benchmark scenario based on the following assumptions. We will choose $p=1$ and $q=10 \mathrm{in} \mathrm{Eq.} \mathrm{(20),} \mathrm{this} \mathrm{implies} m_{6,7} \simeq 10 m_{4,5}$ and $m_{5}-m_{4} \simeq m_{7}-m_{6} \simeq \mu_{s}$. With Regards to the scalar sector, we take $\tan \beta=1$ in Eq. 19] and set all physical neutral and charged scalar masses to be 1 TeV, except for the SM Higgs boson with mass $m_{H^{0}}=125 \mathrm{GeV}$. We span the parameter space in the following way:

- We fix the low-energy neutrino parameters to their best-fit values given by Table 1 , and compute the effective neutrino mass matrix elements defined in the ISS approximation for both neutrino mass spectra (NO and IO). Notice that, the scales $m_{D_{i}}, M$ and 
$\mu_{s}$ are not uniquely defined since $\mathbf{M}_{\mathrm{eff}}$ is invariant under the rescalings

$$
M \rightarrow a M, \mu_{s} \rightarrow b \mu_{s}, m_{D_{i}} \rightarrow \frac{a}{\sqrt{b}} m_{D_{i}}
$$

To probe a wide range of scales we vary $M$ and $\mu_{s}$ in the intervals $\left[1,10^{4}\right] \mathrm{GeV}$ and $\left[1,10^{11}\right]$ eV. For a given pair $\left(M, \mu_{s}\right)$, we set the rescaling parameters with respect to the initial values, namely $a=M / 100$ and $b=\mu_{s} / 10$. Using Eq. (26), we obtain the corresponding $m_{D_{i}}$. Notice that, to ensure perturbativity of the Dirac Yukawa couplings $b_{i}$ we require $y_{\max }=\max \left\{b_{1,2}=\right.$ $\left.m_{D_{1,2}} / v_{1}, b_{3,4}=m_{D_{3,4}} / v_{2}\right\} \leq 5$. We stress that rescaling $M, \mu_{s}$ and $m_{D_{i}}$ is the only way to probe the parameter space of our model since ratios among different $m_{D_{i}}$ are determined by the fixed low-energy parameters.

- For each set of $\left(M, \mu_{s}, m_{D_{i}}\right)$, we define the full $7 \times 7$ neutrino mass matrix $\mathcal{M}$ using Eqs. (1) and [20), and diagonalise it as indicated in Eq. (4) to determine $\mathcal{U}$ and $m_{1-7}$. The active neutrino mixing is characterised by the non-unitary matrix $\mathbf{U}$ of Eq. (13).

Throughout the rest of this work, we use as reference parameters the average mass of the lightest sterile neutrino pair $m_{45}$, a degeneracy parameter $r_{N}$ and the mixing of the electron with the lightest sterile neutrino $V_{e N}$, which are defined as

$$
m_{45}=\frac{m_{4}+m_{5}}{2} \simeq M, r_{N}=\frac{m_{5}-m_{4}}{m_{45}} \simeq \frac{\mu_{s}}{m_{45}}, V_{e N}=\left|\mathbf{B}_{e 4}\right| \simeq \frac{m_{D_{4}}}{\sqrt{2} m_{45}}
$$

The results of our numerical analysis are shown in Fig. 2 for the case $\mathrm{NO}_{e}$ (left panel) and $\mathrm{IO}_{e}$ (right panel). The colour codes in the legend of the upper-left panel apply to the whole figure. By inspecting these plots we conclude that:

- The validity of the inverse-seesaw approximation up to $1 \%$ level, i.e. $\Delta_{\text {ISS }}<1 \%$, imposes lower bounds on the LNV parameter $\mu_{s}>10-20 \mathrm{eV}$ (cyan shaded regions), which correspond to upper bounds on the mixing $V_{e N}^{2} \lesssim 10^{-4}-10^{-3}$. The light (dark) grey regions show that a considerable fraction of the parameter space is excluded if we take into account the perturbativity requirement $b_{i}^{\max }<1(5)$.

- The MEG [23] and SINDRUM II [24] bounds on BR $(\mu \rightarrow e \gamma)$ and $\mathrm{CR}(\mu-e, \mathrm{Au})$ exclude $m_{45} \gtrsim 1-10 \mathrm{GeV}$ for $\Delta_{\text {ISS }} \gtrsim 1 \%$. Moreover, the improvement on $\operatorname{BR}(\mu \rightarrow e \gamma)$ projected by MEG II [25] (solid orange contour) would have a marginal impact in covering our model's the parameter space. On the other hand, reaching a sensitivity of $\mathrm{BR}(\mu \rightarrow 3 e)$ at the $10^{-16}$ level $(\mathrm{Mu} 3 \mathrm{e}[26])$ would be more relevant in constraining the parameter space, especially for heavier sterile neutrinos (for larger $m_{45}$ ).

- The COMET [27] and PRISM/PRIME [28] projected sensitivities for CR $(\mu-e, \mathrm{Al})$ and CR $(\mu-e$, Ti), represented by black and blue dash-dotted contours, respectively, cover a considerable part of the parameter space, however, they leave unprobed the regions in shaded blue where $\mathrm{CR}(\mu-e, \mathrm{Ti})<10^{-18}$. In the best-case scenario $\mathrm{NO}_{e}$, probing $\mathrm{CR}(\mu-e, \mathrm{Ti})$ down to $10^{-18}$ would cover the whole parameter space, as can be seen in the left panel.

\section{CONSTRAINTS ON HEAVY STERILE NEUTRINOS AND FUTURE PROSPECTS}

In this section we analyse the constraints imposed by cLFV experimental searches on our model, from a perspective where $\mu_{s}$ is replaced by the active-sterile mixing parameters $\mathbf{B}_{\alpha j}$. In our framework, we only need to consider one of these quantities since, as seen in Section 5 and through Eq. 24), they are all correlated. From now on, we take as constrained parameters $m_{45}$ and $V_{e N}^{2}$ defined in Eq. 27). We will be able to compare the constraining power of the cLFV processes discussed in the previous section with other experimental searches which are translated into constraints on mass and mixing parameters. We will consider:

- Beam-dump experiments: A beam-dump experiment consists of a primary beam striking a high-density target which produces a large number of secondary heavy mesons that, in the presence of active-sterile mixing, can decay to final states with sterile neutrinos. Current experiments: NA3 [29] and CHARM [30]; Future experiments: SHIP [31] and DUNE [32];

- High-energy colliders: In electron-positron colliders collaborations look for heavy neutrinos $N$ produced via on-shell $Z$ boson decays $e^{+} e^{-} \rightarrow Z \rightarrow N v$. Several subsequent $N$ decay modes are considered, namely $N \rightarrow Z^{*} v\left(Z^{*} \rightarrow \ell \ell, v v, j j\right)$ and $N \rightarrow W^{*} \ell\left(W^{*} \rightarrow \ell v_{\ell}, j j^{\prime}\right)$. In hadron $p p$ colliders collaborations look for $N$ production in $W^{ \pm} \rightarrow \ell^{ \pm} N$ followed by subsequent decays $N \rightarrow W^{ \pm *} \ell^{\mp}\left(W^{ \pm *} \rightarrow \ell^{ \pm} v_{\ell}\right)$ with $\ell=e, \mu$. This leads to trilepton signatures of two types, the first one is the LNV mode with same-sign dileptons of the same flavour (electrons and muons) in the final state and the other one is the lepton number conserving case. Additionally, callaborations have conducted searches for heavy neutrinos produced in the $p p \rightarrow W^{ \pm *} \rightarrow \ell^{ \pm} N$ decay channel into same-sign dileptons and jets $N \rightarrow W^{ \pm} \ell^{ \pm}\left(W^{ \pm} \rightarrow j j^{\prime}\right)$.

Current experiments: L3 [33] and DELPHI [34] from the Large Electron-Positron (LEP) collider; ATLAS [35] and CMS [36] from the LHC.

Furthermore, we will consider constraints arising from the SM Higgs boson $H^{0} \rightarrow N v$ decay channel. The subsequent decays $N \rightarrow \ell W^{*}\left(W^{*} \rightarrow \ell v\right)$ and $N \rightarrow \ell Z^{*}\left(Z^{*} \rightarrow \ell^{+} \ell^{-}\right)$have been studied at the LHC [37.

Future experiments: High-luminosity LHC (HL-LHC) [38], Future Circular Hadron Collider (FCC-hh) [38], Future Circular electron-positron Collider (FCC-ee) [39], $e^{+} e^{-}$linear colliders such as the International Linear Collider (ILC) [40] and Compact Linear Collider (CLIC) [41].

Additionally, detectors placed near LHC interaction points would allow for searches of sterile neutrinos produced in $p p$ collisions through the reconstruction of displaced vertices in a low-background environment. Several proposals have been 

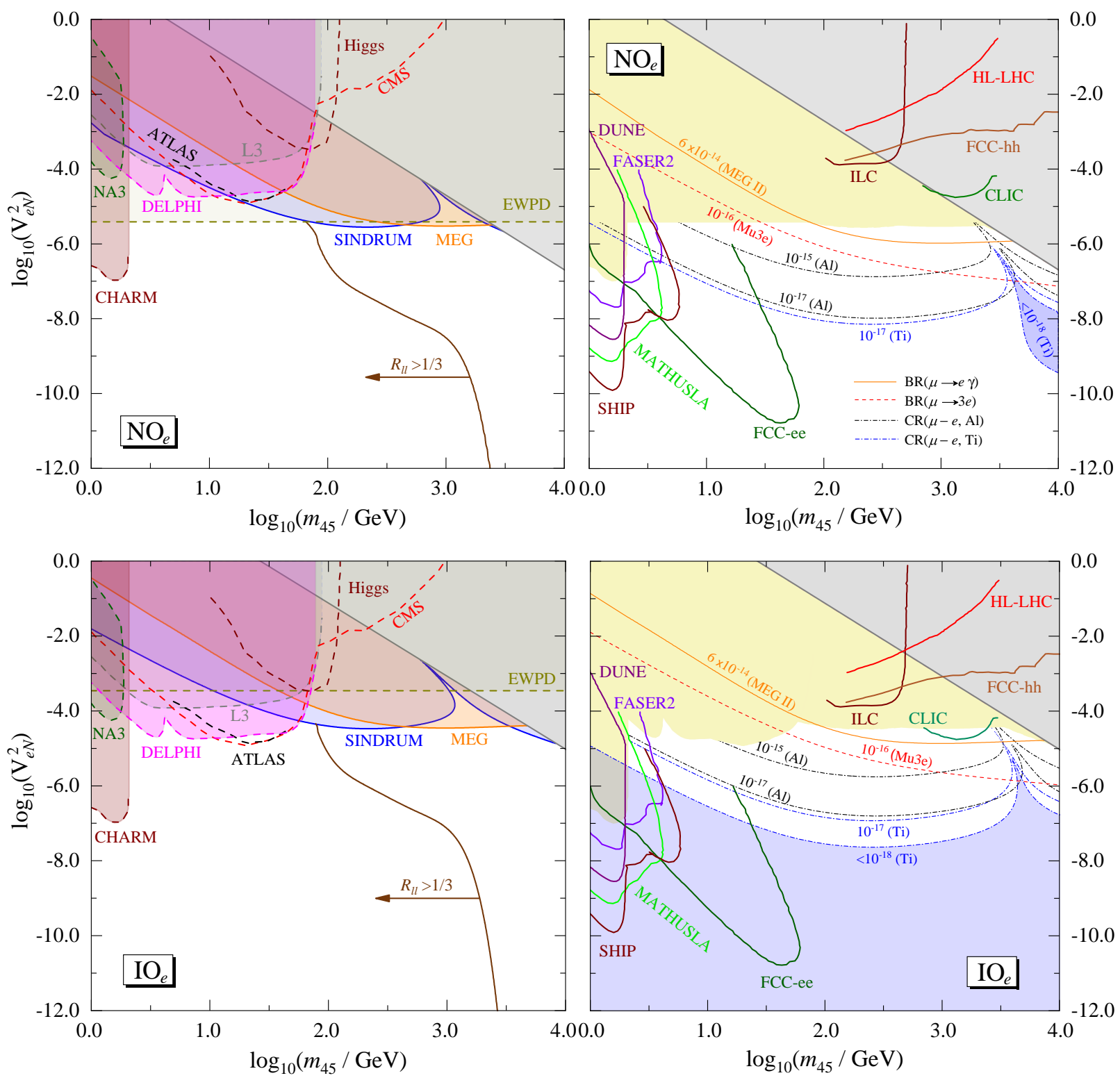

FIGURE 3: [Left] Constraints imposed on the $\left(m_{45}, V_{e N}^{2}\right)$ parameter space by the: MEG and SINDRUM bounds on BR $(\mu \rightarrow e \gamma)$ and $\mathrm{CR}(\mu-e, \mathrm{Au})$ (see Section 6), current searches conducted at colliders, beam-dump experiments as well as EWPD (see discussion in the main text). As in Fig. $2 b_{i}^{\max }>5$ inside the grey-shaded region. $R_{l l}>1 / 3$ to the left of the solid brown line. [Right] Projected sensitivities for future CLFV searches and other experiments discussed in the text. The overlap between the current constraints shown on the left panels are indicated by the yellow-shaded regions. Within the blue shaded region we have $\mathrm{CR}(\mu-e, \mathrm{Ti})<10^{-18}$. The top (bottom) panels correspond to the $\mathrm{NO}_{e}\left(\mathrm{IO}_{e}\right)$ case.

put forward to conduct this kind of analyses, namely we will consider the projected sensitivies from the FASER2 [42] and MATHUSLA [43] experiments.

Lastly, we will use the criterion $R_{l l} \geq 1 / 3$ to identify the regions of the parameter space where LNV decays are unsuppressed [44].

- Electroweak precision data (EWPD): The presence of sterile neutrinos lead to a non-unitary mixing matrix U [cf. Eq. [13]. Deviations from unitarity are constrained by neutrino oscillation data, electroweak precision tests and LFV decays. In fact, the off-diagonal elements of $\eta_{\alpha \beta}$ defined in Eq. (15) are restricted by the cLFV decays studied in the previous section. The diagonal elements $\eta_{\alpha \alpha}$ are restricted by SM gauge boson decays, namely $W \rightarrow \ell_{\alpha} v_{\alpha}$ and $Z \rightarrow v v$, as well as, universality tests in $W$ and $\pi$ decays. We will use the bounds on $\eta_{\alpha \beta}$ from Ref. [45] and we are able thanks to Eqs [15] and [27) to translate them into bounds on $V_{e N}^{2}=\left|\mathbf{B}_{e 4}\right|^{2}$. 
In the left panels of Fig. 3are presented all the current constraints mentioned above, together with those stemming from $\mu \rightarrow e \gamma$ (MEG) and $\mu-e$ conversion in Au (SINDRUM) searches (see Fig. 2), now shown in the $\left(m_{45}, V_{e N}^{2}\right)$ plane. For the EWPD exclusion regions we consider the most restrictive $V_{e N}^{2}$ limits extracted from $\left|\eta_{\mu \mu}\right|$. On the right panels, the projected sensitivities of future experiments cited above are shown, including the cLFV ones already presented in Fig. 2 . now in the $\left(m_{45}, \mu_{s}\right)$ plane. The overlap of the current exclusion regions (left panels) is shown in light yellow. Looking at the figure we conclude that:

- For sterile neutrino masses $m_{45} \gtrsim 2 \mathrm{GeV}$, the strongest constraints are (typically) those imposed by the SINDRUM and MEG bounds on $\mathrm{BR}(\mu \rightarrow e \gamma)$ and $\mathrm{CR}(\mu-e, \mathrm{Au})$, respectively, and by EWPD (left panels) for the $\mathrm{NO}_{e}$. However, in the $\mathrm{IO}_{e}$ case, for $2 \mathrm{GeV} \lesssim m_{45} \lesssim 50 \mathrm{GeV}$, the DELPHI, ATLAS and CMS limits are stronger. In both cases, the CHARM exclusion region is more constraining when $m_{45}=1-2 \mathrm{GeV}$. Also, the EWPD exclusion regions will not be the same for the other $\mathrm{NO}_{\mu, \tau}$ and IO $\mathrm{IO}_{\mu, \tau} \mathrm{scenarios}$ since the $\mathrm{U}(1)$ flavour symmetries, together with present neutrino data, impose different relations among the $\mathbf{B}_{\alpha j}$.

- Any signal of sterile neutrinos for $V_{e N}^{2} \gtrsim 10^{-4}$ at future hadron or linear colliders (HL-LHC, FCC-hh, CLIC and ILC regions) would not be compatible with the limits already imposed by current constraints from cLFV searches and EWPD (see right panels). Therefore, high-energy collider probes conducted at the FCC-ee and at experiments like SHIP, MATHUSLA, DUNE and FASER2 turn out to be of utmost importance in our framework.

- For $\mathrm{NO}_{e}, \mathrm{cLFV}$ indirect searches are fully complementary to the aforementioned direct ones, this is not the case for $\mathrm{IO}_{e}$ nor for the remaining scenarios (see Ref. [13] for details). In particular, for IO neutrino masses, the region with $V_{e N}^{2} \lesssim 10^{-9}-10^{-8}$ cannot be probed by future $\mu-e$ conversion experiments. However, such mixing regimes can be covered by displaced-vertex experiments and by a high-luminosity $Z$ factory like the FCC-ee. Notice that $R_{l l} \geq 1 / 3$ within the sensitivity regions of those searches (see the brown solid lines in the left panels), indicates that LNV sterile neutrino decays are not suppressed. Furthermore, in the absence of a positive $\mu \rightarrow e \gamma$ signal, the impact of MEG II data would be mild. Instead, if that decay is observed, we can set relatively narrow ranges for $m_{45}$ and $V_{e N}^{2}$. For $\mu \rightarrow 3 e$, future probes conducted by the Mu3e collaboration will be able to probe $V_{e N}^{2}$ down to $10^{-6}-10^{-7}$ for a wide range of sterile neutrino masses.

\section{CONCLUDING REMARKS}

We have investigated the minimal ISS mechanism with couplings constrained by U(1) flavour symmetries, and with all fermion masses generated through SSB by the VEVs of doublet and singlet scalar fields. After finding the maximally-restrictive mass matrices that are compatible with current neutrino data, we identified all possible $\mathrm{U}(1)$ symmetry realisations and concluded that in order to implement those symmetries we needed at least two Higgs doublets and two complex scalar singlets.

The presence of such singlets opens up the possibility for SCPV, which is successfully communicated to the lepton sector via their couplings to the new sterile fermions. As a result of SCPV and the Abelian symmetries, the low-energy Majorana and Dirac $\mathrm{CP}$ phases are correlated. Due to the flavour symmetries, the heavy-light mixings are not independent, being their ratios entirely determined by the lepton sector observables. This provides a very constrained setup for phenomenological studies.

We have analysed several cLFV decays and obtained the exclusion regions set by the experimental limits on $\mathrm{BR}(\mu \rightarrow e \gamma)$ and $\operatorname{CR}(\mu-e, \mathrm{Au})$ which establish upper bounds on $V_{e N}^{2}$ of about $10^{-4}-10^{-5}$. We discussed the prospects to further explore the parameter space in view of the projected sensitivities of future cLFV searches, namely those dedicated to $\mu \rightarrow e \gamma, \mu \rightarrow 3 e$ and $\mu-e$ conversion in nuclei.

After analysing the constraining power of cLFV processes, we focused on alternative probes, namely collider and beam-dump experimental searches which are sensitive to sterile neutrinos. We concluded that the HL-LHC, FCC-hh, ILC and CLIC sensitivity regions are already excluded by current CLFV and EWPD constraints. On the other hand, searches at the FCC-ee and at experiments like SHIP, MATHUSLA and FASER2 would be highly complementary to the Mu3e, COMET and PRISM/PRIME projects. Hence, it is clear that a single positive signal in any of those experiments would put at test the scenarios studied in this work. In this sense, further symmetry-motivated studies performed in the context of sterile neutrino searches are needed moving forward.

\section{ACKNOWLEDGEMENTS}

This work has been supported by "Fundação para a Ciência e a Tecnologia" (FCT, Portugal) through the projects UIDB/00777/2020, UIDP/00777/2020, CERN/FIS-PAR/0004/2019, and PTDC/FIS-PAR/29436/2017.

\section{References}

[1] P. Minkowski, Phys. Lett. 67B (1977) 421.

[2] M. Gell-Mann, P. Ramond and R. Slansky, Conf. Proc. C790927 (1979) 315 [arXiv:1306.4669 [hep-th]].

[3] J. Schechter and J. W. F. Valle, Phys. Rev. D 22 (1980) 2227.

[4] S. L. Glashow, NATO Sci. Ser. B 61 (1980) 687.

[5] R.N. Mohapatra, Phys. Rev. Lett. 56 (1986) 561.

[6] R. N. Mohapatra and J. W. F. Valle, Phys. Rev. D 34, 1642 (1986)

[7] M.C. Gonzalez-Garcia and J.W.F. Valle, Phys. Lett. B 216 (1989) 360.

[8] R. Gonzalez Felipe and H. Serodio, J. Phys. G 44 (2017) no.6, 065002 [arXiv:1604.06724 [hep-ph]].

[9] S. S. Correia, R. G. Felipe and F. R. Joaquim, Phys. Rev. D 100 (2019) no.11, 115008 [arXiv:1909.00833 [hep-ph]].

[10] C. I. Low and R. R. Volkas, Phys. Rev. D 68 (2003), 033007 [arXiv:hep-ph/ 0305243 [hep-ph]].

[11] G. C. Branco, P. M. Ferreira, L. Lavoura, M. N. Rebelo, M. Sher and J. P. Silva, Phys. Rept. 516 (2012), 1-102 [arXiv:1106.0034 [hep-ph]]. 
[12] A. Abada and M. Lucente, Nucl. Phys. B 885 (2014), 651-678 [arXiv:1401.1507 [hep-ph]].

[13] H. B. Camara, R. G. Felipe and F. R. Joaquim, [arXiv:2012.04557 [hep-ph]].

[14] G. 't Hooft, NATO Sci. Ser. B 59, 135-157 (1980)

[15] W. Grimus and L. Lavoura, JHEP 11 (2000), 042 [arXiv:hep-ph/0008179 [hep-ph]].

[16] W. Rodejohann and J. W. F. Valle, Phys. Rev. D 84, 073011 (2011) [arXiv:1108.3484 [hep-ph]].

[17] P. A. Zyla et al. [Particle Data Group], PTEP 2020 (2020) no.8, $083 \mathrm{C} 01$

[18] P. F. de Salas, D. V. Forero, S. Gariazzo, P. Martínez-Miravé, O. Mena, C. A. Ternes, M. Tórtola and J. W. F. Valle, JHEP 02 (2021), 071 [arXiv:2006.11237 [hep-ph]].

[19] P. O. Ludl and W. Grimus, JHEP 07 (2014), 090 [erratum: JHEP 10 (2014), 126] [arXiv:1406.3546 [hep-ph]].

[20] H. Serôdio, Phys. Rev. D 88 (2013) no.5, 056015 [arXiv:1307.4773 [hep-ph]].

[21] I. P. Ivanov and C. C. Nishi, JHEP 11 (2013), 069 [arXiv:1309.3682 [hep-ph]].

[22] G. C. Branco, R. G. Felipe and F. R. Joaquim, Rev. Mod. Phys. 84 (2012), 515-565 [arXiv:1111.5332 [hep-ph]].

[23] A. M. Baldini et al. [MEG], Eur. Phys. J. C 76 (2016) no.8, 434 [arXiv:1605.05081 [hep-ex]].

[24] W. H. Bertl et al. [SINDRUM II], Eur. Phys. J. C 47 (2006), 337-346

[25] A. M. Baldini et al. [MEG II], Eur. Phys. J. C 78 (2018) no.5, 380 [arXiv:1801.04688 [physics.ins-det]].

[26] A. Blondel, A. Bravar, M. Pohl, S. Bachmann, N. Berger, M. Kiehn, A. Schoning, D. Wiedner, B. Windelband and P. Eckert, et al. [arXiv:1301.6113 [physics.ins-det]].

[27] R. Abramishvili et al. [COMET], PTEP 2020 (2020) no.3, 033C01 [arXiv:1812.09018 [physics.ins-det]].

[28] A. Alekou, R. Appleby, M. Aslaninejad, R. J. Barlow, R. C. K. M. Hock, J. Garland, L. J. Jenner, D. J. Kelliher, Y. Kuno and A. Kurup, et al. [arXiv:1310.0804 [physics.acc-ph]].

[29] J. Badier et al. [NA3], Z. Phys. C 31 (1986), 21

[30] F. Bergsma et al. [CHARM], Phys. Lett. B 166 (1986), 473-478

[31] C. Ahdida et al. [SHiP], JHEP 04 (2019), 077 [arXiv:1811.00930 [hep-ph]].

[32] I. Krasnov, Phys. Rev. D 100 (2019) no.7, 075023 [arXiv:1902.06099 [hep-ph]].

[33] O. Adriani et al. [L3], Phys. Lett. B 295 (1992), 371-382

[34] P. Abreu et al. [DELPHI], Z. Phys. C 74 (1997), 57-71 [erratum: Z. Phys. C 75 (1997), 580]

[35] G. Aad et al. [ATLAS], JHEP 10 (2019), 265 [arXiv:1905.09787 [hep-ex]].

[36] A. M. Sirunyan et al. [CMS], Phys. Rev. Lett. 120 (2018) no.22, 221801 [arXiv:1802.02965 [hep-ex]].

[37] A. Das, P. S. B. Dev and C. S. Kim, Phys. Rev. D 95 (2017) no.11, 115013 [arXiv:1704.00880 [hep-ph]].

[38] S. Pascoli, R. Ruiz and C. Weiland, JHEP 06 (2019), 049 [arXiv:1812.08750 [hep-ph]].

[39] A. Blondel et al. [FCC-ee study Team], Nucl. Part. Phys. Proc. 273-275 (2016), 1883-1890 [arXiv:1411.5230 [hep-ex]].

[40] S. Banerjee, P. S. B. Dev, A. Ibarra, T. Mandal and M. Mitra, Phys. Rev. D 92 (2015), 075002 [arXiv:1503.05491 [hep-ph]].

[41] A. Das, S. Jana, S. Mandal and S. Nandi, Phys. Rev. D 99 (2019) no.5, 055030 [arXiv:1811.04291 [hep-ph]].

[42] F. Kling and S. Trojanowski, Phys. Rev. D 97 (2018) no.9, 095016 [arXiv:1801.08947 [hep-ph]].

[43] D. Curtin, M. Drewes, M. McCullough, P. Meade, R. N. Mohapatra, J. Shelton, B. Shuve, E. Accomando, C. Alpigiani and S. Antusch, et al. Rept. Prog. Phys. 82 (2019) no.11, 116201 [arXiv:1806.07396 [hep-ph]].

[44] M. Drewes, J. Klarić and P. Klose, JHEP 11 (2019), 032 [arXiv:1907.13034 [hep-ph]].

[45] E. Fernandez-Martinez, J. Hernandez-Garcia and J. Lopez-Pavon, JHEP 08 (2016), 033 [arXiv:1605.08774 [hep-ph]]. 